Check for updates

Cite this: RSC Adv., 2019, 9, 20663

\title{
Ag-NPs/MWCNT composite-modified silver-epoxy paste with improved thermal conductivity
}

\author{
Yanchao Li, (D) Guoyou Gan, ${ }^{*}$ Yukuan Huang, Xianglei Yu, Junhua Cheng \\ and Chengbin Liu
}

Heat dissipation is a critical issue in high-performance electronics, which needs to be solved, and an electronic paste is a good choice to solve this issue. In this paper, silver nanoparticles/multi-walled carbon nanotube (Ag-NPs/MWCNT) composites were prepared by the chemical process for the modification of electronic pastes. The micromorphology and spectral analysis of the as-prepared AgNPs/MWCNT composites indicated that Ag-NPs were uniformly distributed on the MWCNT surfaces with high distribution densities; the average size of Ag-NPs was estimated to be $8.29 \mathrm{~nm}$. The as-obtained Ag-NPs/MWCNT composites were then added to a silver-epoxy paste. Field emission scanning electron microscopy (FESEM), thermogravimetry (TG) and thermal conductivity analyses suggested the incorporation of Ag-NPs/MWCNT composites in the silver-epoxy paste; Ag flakes were better connected by Ag-NPs/MWCNTs, which improved the thermal conductivity of the paste from 0.73 to $0.96 \mathrm{~W} \mathrm{~m}^{-1}$ $\mathrm{K}^{-1}$. However, more weight loss was observed when Ag-NPs/MWCNTs were incorporated in the silverepoxy paste. Overall, the addition of Ag-NPs/MWCNTs into the silver-epoxy paste increased the thermal conductivity, which can be applied to high-performance electronics.

Received 25th April 2019

Accepted 17th June 2019

DOI: $10.1039 / \mathrm{c} 9 \mathrm{ra03090e}$

rsc.li/rsc-advances dimensional architectures have better characteristics in terms of increased specific surface areas, high tensile strength and toughness, good thermal conductivity $\left(3000 \mathrm{~W} \mathrm{~m}^{-1} \mathrm{~K}^{-1}\right)$, and excellent electrical conductivity. Hence, MWCNTs have attracted wide attention in both research and application fields, especially for microelectronics and composite materials. ${ }^{\mathbf{1 2 - 1 6}}$ In addition, the deposition of metallic silver on MWCNT surfaces can further improve their performances due to their high thermal and electrical conductivities. ${ }^{17-20}$ Better interaction between Ag-NPs and MWCNTs might be achieved by strong covalent bonds or weak intermolecular bonds, such as hydrophobic interactions, hydrogen bonding or electrostatic attractions. Furthermore, synergetic effects might be created in AgNP-modified MWCNTs, which might improve both the electrical and thermal conductivities as well as enhance the toughening effect. ${ }^{21,22}$ Suh D. et $a .^{3}{ }^{3}$ studied the electrical and thermal properties of TIMs; the addition of Ag-MWCNTs increased the electrical conductivity of the silver-epoxy paste from $1.12 \times 10^{4} \mathrm{~S} \mathrm{~m}^{-1}$ to $1.79 \times 10^{6} \mathrm{~S} \mathrm{~m}^{-1}$, and the thermal conductivity also improved significantly. Some studies have shown that silver nanoparticles/multi-walled carbon nanotube (Ag-NPs/MWCNT) composites are ideal materials for achieving better heat transfer and high thermal conductivity. Note that heat transfer is mainly determined by phonon thermal conductivity, and thermal conductivity includes both electronic thermal conductivity and phonon thermal conductivity. The combination of Ag-NPs/MWCNT composites and $\mathrm{Ag}$ flakes forms three-dimensional heat-conducting structures by low- 
temperature sintering bonding. This process reduces phonon scattering at the interfaces, achieving efficient heat conduction and superior thermal conductivity. ${ }^{3,23}$ However, the thermogravimetry (TG) and morphology of Ag-NPs/MWCNT-modified silver-epoxy composites have not been studied in detail. We expect that the Ag-NPs/MWCNT composites can have better wetting and dispersing properties in the paste system, and more Ag-NPs/MWCNT bridges can be formed between the Ag flakes, with less thermal weight loss and significantly improved thermal conductivity.

In this study, MWCNTs were functionalized by ball milling in the presence of ammonium bicarbonate $\left(\mathrm{NH}_{4} \mathrm{HCO}_{3}\right)$ to yield modified MWCNTs. ${ }^{24}$ Due to the hydrophobicity of MWCNTs and their tendency to self-aggregate by strong van der Waals forces, sodium dodecyl benzene sulfonate (SDBS) was added as a dispersant to form specific directional $\pi-\pi$ interactions with the MWCNT graphite layer via its benzene ring structure; this induced better dispersion of MWCNTs to yield a MWCNT nanofluid with long-term stability. ${ }^{25-30}$ Next, silver ions were adsorbed onto the modified MWCNT surfaces through the $\mathrm{NH}_{2}$ functional groups in the presence of the reducing agent ascorbic acid $\left(\mathrm{C}_{6} \mathrm{H}_{8} \mathrm{O}_{6}\right)$ to deposit Ag-NPs onto the modified MWCNTs (denoted as Ag-NPs/MWCNT composites). The presence of Ag-NPs on the MWCNT surfaces could reduce the van der Waals forces between MWCNTs and improve their dispersion while maintaining the low-temperature sintering activity responsible for metal-metal bonding. Moreover, non-covalent modification did not damage the free electronic structures of MWCNTs, leading to excellent electrical and thermal properties.

In this paper, Ag-NPs/MWCNT composites were prepared by simple chemical methods and studied in detail. The asprepared Ag-NPs/MWCNT composites were then added to a silver-epoxy paste to study their effects on the morphology, TG and thermal conductivity of the silver-epoxy paste.

\section{Experimental}

\subsection{Materials}

MWCNTs (purity $>95 \%$, inner diameter 2-5 $\mathrm{nm}$, outer diameter $<8 \mathrm{~nm}$, length 10-30 $\mu \mathrm{m}$ ) and SDBS (95\%, mixture) were obtained from Sinopharm Chemical Reagent Co. Ltd. Ammonium bicarbonate $\left(\mathrm{NH}_{4} \mathrm{HCO}_{3}, \mathrm{AR}\right)$ was purchased from Tianjin Fenchuan Chemical Reagent Technology Co. Ltd. Acetone $\left(\mathrm{C}_{3} \mathrm{H}_{6} \mathrm{O}\right.$, AR) was provided by Chengdu Kelong Chemical Reagent Factory. Silver nitrate $\left(\mathrm{AgNO}_{3}, \mathrm{AR}\right)$ was purchased from Shanghai Fine Chemical Materials Research Institute, and ascorbic acid $\left(\mathrm{C}_{6} \mathrm{H}_{8} \mathrm{O}_{6}, \mathrm{AR}\right)$ was acquired from Sinopharm Chemical Reagent Co. Ltd. Ag flakes and the bisphenol A epoxy resin were produced by Kunming Institute of Precious Metals.

\subsection{Preparation of Ag-NPs/MWCNT composites}

The flow chart for the preparation of Ag-NPs/MWCNT composites is shown in Fig. 1. MWCNTs were functionalized using the ball milling technology in the presence of ammonium bicarbonate $\left(\mathrm{NH}_{4} \mathrm{HCO}_{3}\right)$. Briefly, $0.1 \mathrm{~g}$ MWCNTs and $2.0 \mathrm{~g}$
$\mathrm{NH}_{4} \mathrm{HCO}_{3}$ were added into a ball milling container, and $\mathrm{C}_{3} \mathrm{H}_{6} \mathrm{O}$ solution was then added to the ball milling container to yield a homogenous mixture, which was rolled at $300 \mathrm{rpm}$ for $6 \mathrm{~h}$. A total of three ball milling containers containing 2, 20 and 100 pieces of $\mathrm{ZrO}_{2}$ milling beads with the corresponding diameters of 15, 7 and $4 \mathrm{~mm}$ were prepared. After ball milling, the products were poured into test tubes and washed 3 times with deionized water and ethanol to remove residual impurities. Next, the functionalized MWCNTs were added to a SDBS solution and then ultrasonically dispersed for $2 \mathrm{~h}$ in an ice bath. The obtained suspensions were added to an $\mathrm{AgNO}_{3}$ solution under constant magnetic stirring at room temperature for $6 \mathrm{~h}^{31}$ Afterwards, a solution of the reducing agent $\mathrm{C}_{6} \mathrm{H}_{8} \mathrm{O}_{6}$ (0.1 $\left.\mathrm{mol} \mathrm{L}^{-1}\right)$ was added dropwise to the above system and magnetically stirred for $0.5 \mathrm{~h}$. After standing for about $6 \mathrm{~h}$, the Ag-NPs/MWCNT composites were collected by centrifugation, and the residual impurities were removed by washing several times with ethanol. ${ }^{26}$

\subsection{Preparation of silver-epoxy paste with modified Ag-NPs/ MWCNT composites}

The total weight of the silver-epoxy paste was set to $20 \mathrm{~g}$ and that of the Ag flakes was kept at $60 \mathrm{wt} \%$. The specific preparation steps consisted of first adding the premixed Ag flakes and bisphenol A epoxy resin into the paste. The as-prepared Ag-NPs/ MWCNT composites were then added, and the mixture was dispersed using a three-roll mill. Finally, defoaming was performed under reduced pressure to yield the modified electronic paste. The paste was printed on a high-temperature-resistant polyester film (PET) by screen printing, sintered in an oven at $150{ }^{\circ} \mathrm{C}$ for $2 \mathrm{~h}$, and then taken out and cooled to room temperature. As a control experiment, silver-epoxy paste was prepared according to the same method but we added pristine MWCNTs; it was compared with the pure silver-epoxy paste. Meanwhile the weights of both the pristine MWCNTs and AgNPs/MWCNT composites were kept the same at $1 \mathrm{wt} \%$ of the total weight.

\subsection{Characterization}

The micromorphologies of the Ag-NPs/MWCNT composites were examined by transmission electron microscopy (TEM, JEM-2100, Japan). Inductively coupled plasma optical emission spectrometry (ICP-OES, Prodigy, America) was used to measure the silver content of the Ag-NPs/MWCNT composites. Fourier transform infrared (FT-IR, Bruker ALPHA, Germany) spectroscopy was utilized to gain a better understanding of the chemical bonding. Powder X-ray diffraction (XRD, D8-Advance, Germany) was used for crystal structure identification. Raman spectroscopy of the Ag-NPs/MWCNT composites was performed using a Raman spectrometer (LabRAM HR Evolution, France). Field emission scanning electron microscopy (FESEM, XL30ESEMTEP, Netherlands) was used to identify the dispersibility and wettability of the MWCNTs in the epoxy matrix. A synchronous differential thermal analyzer (STA-409PC, Germany) was employed for TG analysis. A hot disk thermal conductivity meter 


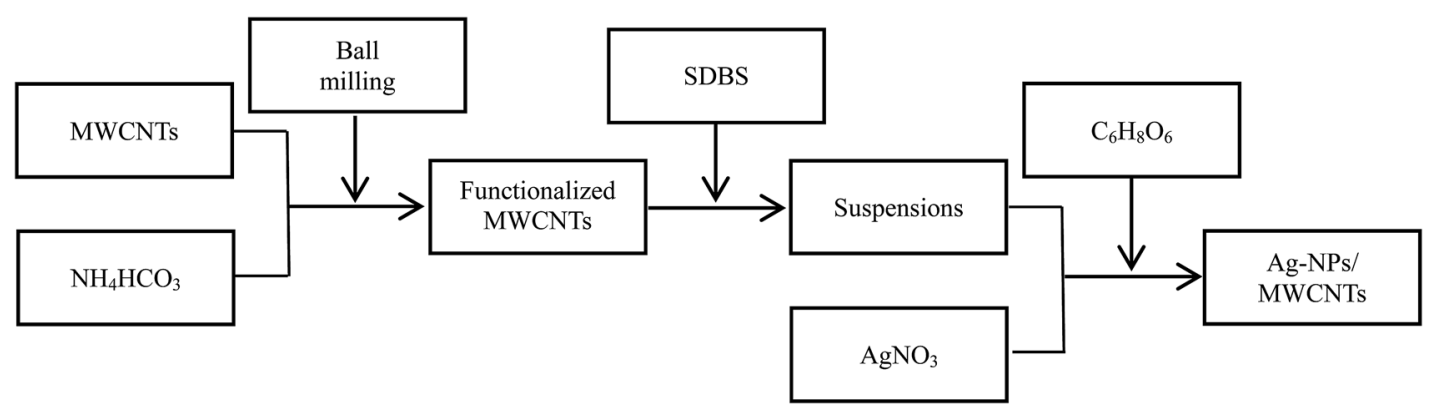

Fig. 1 Flow chart for the preparation of Ag-NPs/MWCNT composites.

(TPS 2500, Sweden) was utilized for the thermal conductivity measurements.

\section{Results and discussion}

\subsection{Morphology and structure of the Ag-NPs/MWCNT composites}

The morphologies of the as-obtained Ag-NPs/MWCNT composites are shown in Fig. 2. The TEM images of the AgNPS/MWCNT composites obtained by ball milling of MWCNTs with SDBS as a dispersant are depicted in Fig. 2(a). AgNPs were clearly observed on the MWCNT surfaces, with an average size of $8.29 \mathrm{~nm}$ (Fig. 2(b)). In addition, different forms of Ag-NPs were uniformly distributed on the MWCNT surfaces at high distribution densities without aggregation; some Ag-NPs were dispersed around MWCNTs, which was probably due to the uniform nucleation of NPs. In addition, using ICP-OES, the silver content of Ag-NPs/MWCNT composites was found to be $45.71 \%$.

Fig. 3 presents the FT-IR spectra of pristine MWCNTs, functionalized MWCNTs, and Ag-NPs/MWCNT composites. The vibration at around $1640 \mathrm{~cm}^{-1}$ in all spectra indicates a $\mathrm{C}=\mathrm{C}$ tensile mode. The peak around $1397 \mathrm{~cm}^{-1}$ originates from the vibration of $\mathrm{C}-\mathrm{H}$ groups. The peak at $3244.05 \mathrm{~cm}^{-1}$ for the functionalized MWCNTs and that at $3442.58 \mathrm{~cm}^{-1}$ for the AgNPs/MWCNT composites were attributed to the vibration of $\mathrm{NH}_{2}$ groups. All these data confirmed the successful introduction of $\mathrm{NH}_{2}$ groups into the MWCNT surfaces after ball milling of MWCNTs in the presence of $\mathrm{NH}_{4} \mathrm{HCO}_{3}$. This modification induced changes in pristine MWCNT surfaces, which acquired an inert state, becoming a nucleation matrix for silver. Ag-NPs were reduced on the functionalized MWCNT surfaces but the interaction between the $\mathrm{NH}_{2}$ groups and MWCNTs remained.

XRD was used to characterize the formation of Ag crystals; the XRD patterns of pristine MWCNTs, functionalized MWCNTs and Ag-NPs/MWCNT composites are shown in Fig. 4. The Ag-NPs/MWCNT composites with $\mathrm{NH}_{2}$ groups corresponded to the four major crystal planes of $\mathrm{Ag}$ (004), $\mathrm{Ag}$ (103), $\mathrm{Ag}$ (110) and Ag (201), confirming the successful modification of the MWCNT surfaces by Ag-NPs. Also, the carbon peak intensities of MWCNTs were enhanced by ball milling but without inducing damage to the crystal structure of MWCNTs. The presence of $\mathrm{NH}_{2}$ groups on the MWCNT surfaces could improve the connection of Ag-NPs to the MWCNT surfaces.

Raman spectroscopy is useful for characterizing the interaction between MWCNTs and Ag-NPs. Fig. 5 shows the Raman spectra of original MWCNTs, functionalized MWCNTs, and Ag-NPs/MWCNT composites. All peaks are depicted, including the D-band, G-band, G'-band, and D + G bands. The D-band is attributed to the vibration mode derived from the disordered structure of graphite, while the $\mathrm{G}$-band is assigned to the tangential radial mode of the $\mathrm{sp}^{2}$ bonded carbon atoms in graphite. The intensity ratio of the D to $\mathrm{G}$ bands $\left(I_{\mathrm{D}} / I_{\mathrm{G}}\right)$ can be used to evaluate the graphitization degree of MWCNTs. The D and G bands of the Ag-NPs/ MWCNT composites were estimated to be 1337 and
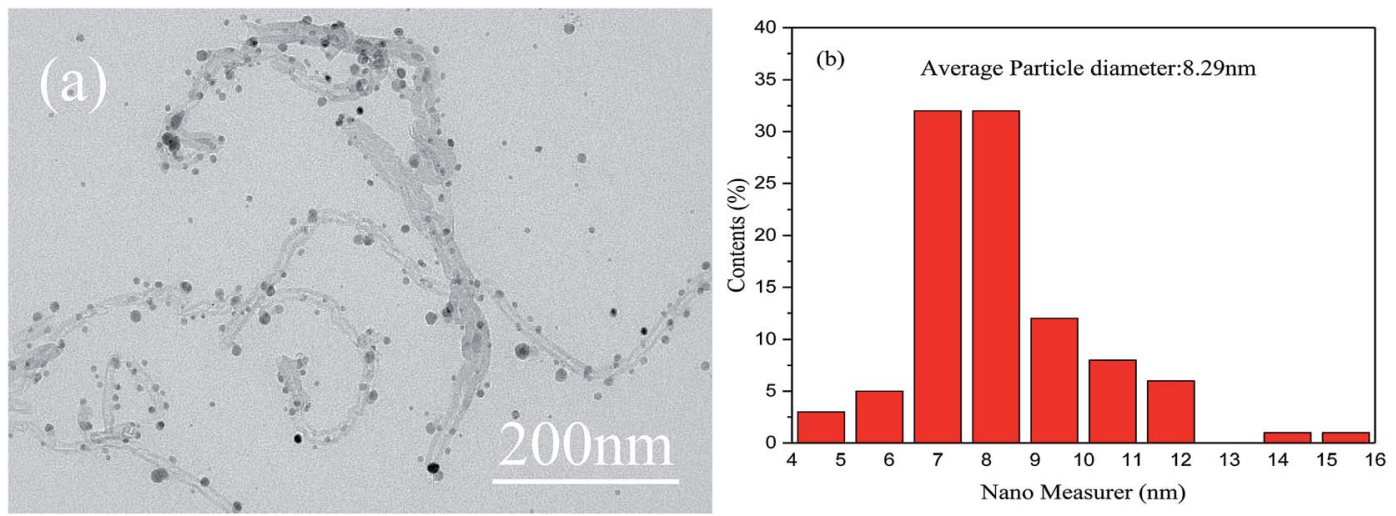

Fig. 2 (a) TEM images of Ag-NPs/MWCNT composites. (b) Size distribution of silver particles. 


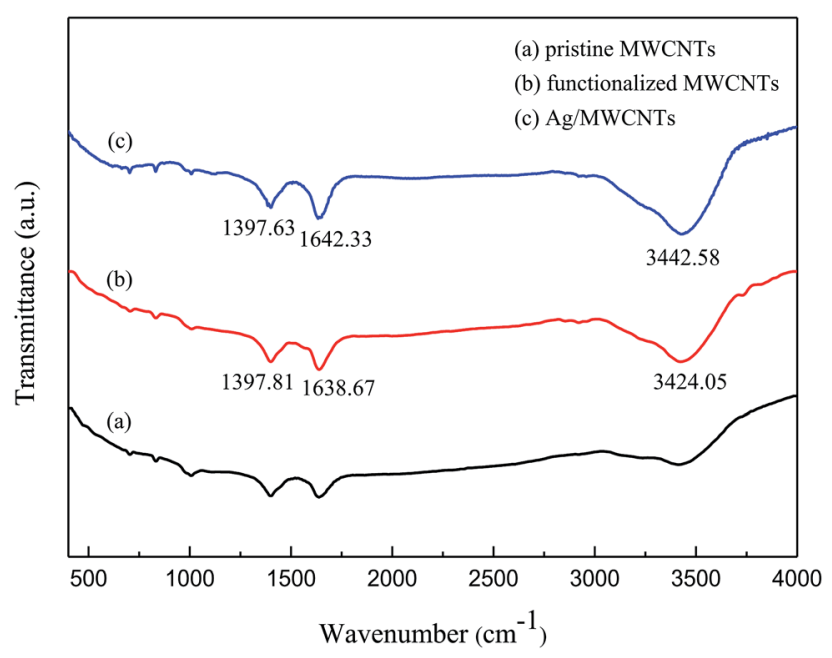

Fig. 3 FT-IR spectra of (a) pristine MWCNTs, (b) functionalized MWCNTs, and (c) Ag-NPs/MWCNT composites.

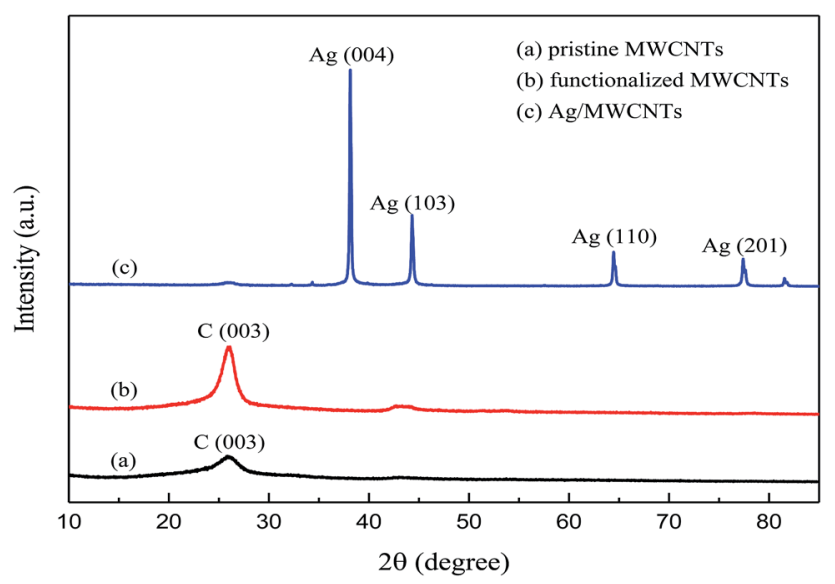

Fig. 4 XRD patterns of (a) pristine MWCNTs, (b) functionalized MWCNTs, and (c) Ag-NPs/MWCNT composites.

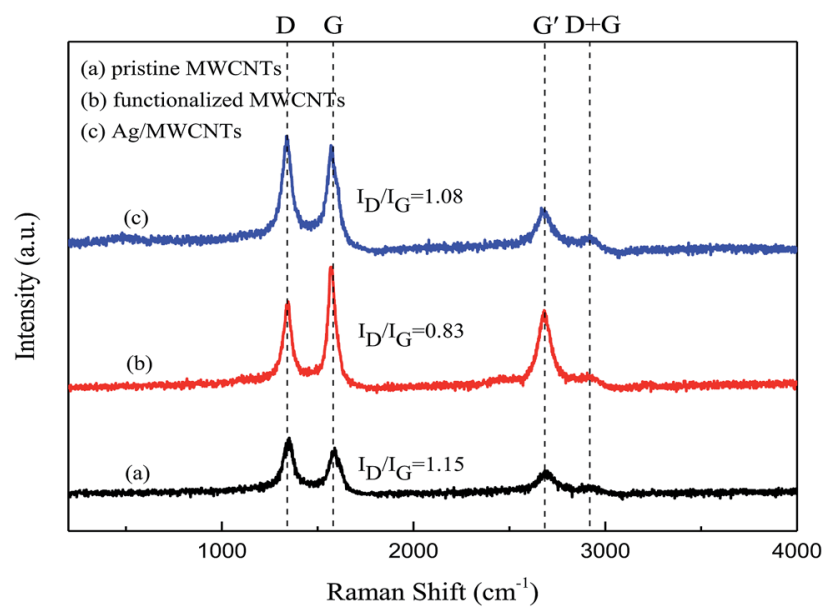

Fig. 5 Raman spectra of (a) pristine MWCNTs, (b) functionalized MWCNTs, and (c) Ag-NPs/MWCNT composites.
$1569 \mathrm{~cm}^{-1}$, respectively. During modification of Ag-NPs, obvious shifts in the $\mathrm{D}$ band to high wavenumbers were noticed, suggesting the occurrence of charge transfer between MWCNTs and Ag-NPs. Thus, the structure of the MWCNT surface changed, and its covalent functionalization introduced $\mathrm{NH}_{2}$ groups, which increased the surface defect density of MWCNTs. The G'-band and D + G bands of the Ag/ MWCNT composites were recorded at 2666 and $2918 \mathrm{~cm}^{-1}$, respectively. The $I_{\mathrm{D}} / I_{\mathrm{G}}$ values of the functionalized MWCNTs appeared smaller than those of the original MWCNTs, meaning that the structure of the MWCNT surfaces became more ordered after ball milling.

\subsection{Analysis of modified silver-epoxy pastes}

Two different MWCNTs were added to prepare silver-epoxy pastes. In the first group, pristine MWCNTs were used as additives (Fig. 6(a and b)). In the second group, Ag-NPs/ MWCNT composites were used as additives (Fig. 6(c and d)). The FESEM images revealed MWCNTs with good wettability that were easily dispersed in the paste system without agglomeration. However, we could not observe AgNPs on the MWCNT surface clearly in Fig. 6(c and d) because the average size of Ag-NPs was about $8.29 \mathrm{~nm}$, and they could not be clearly observed under micron multiples; meanwhile, MWCNTs became bright due to electrical conduction.

In Fig. 6(a and b), the MWCNT content in the paste appears to be low; the dispersion in the paste system was not obvious, and MWCNTs appeared to be overlapped with the Ag flakes. By comparison, Ag-NPs/MWCNTs looked more abundant in the paste with better dispersion (Fig. 6(c and d)). In addition, the Ag flakes were better connected by AgNPs/MWCNTs. This was attributed to decline in the van der Waals forces between MWCNTs as well as improved wettability and dispersity of MWCNTs in the epoxy matrix due to the reduced Ag-NPs on the MWCNT surfaces. Meanwhile, Ag-NPs supported on the MWCNT surfaces exhibited higher sintering activities, which could reinforce interfacial bonding with the $\mathrm{Ag}$ flakes in the paste to form metal-metal contact. Also, large numbers of conduction paths were established between the Ag flakes.

The thermal decomposition of the material was studied by TG analysis through the determination of weight loss. Fig. 7(a-c) display the TG curves of the silver-epoxy paste with added MWCNTs, pristine silver-epoxy paste, and silverepoxy paste with added Ag-NPs/MWCNT composites. All TG curves depicted thermally stable behaviors from 25 to $100{ }^{\circ} \mathrm{C}$ with no significant weight loss. The reason behind the small weight loss was the removal of water at ambient temperatures. The change in weight loss became more obvious at temperatures between 200 and $500{ }^{\circ} \mathrm{C}$. In Fig. 7(a), significant weight loss is recorded starting from $322.0{ }^{\circ} \mathrm{C}$, with a residual mass of $97.800 \%$. At $599.3{ }^{\circ} \mathrm{C}$, the residual mass was $86.956 \%$ and the mass change was $10.845 \%$. In Fig. 7 (b), a significant weight loss is recorded starting from $314.0{ }^{\circ} \mathrm{C}$, with a corresponding residual mass of $98.324 \%$. At $599.7^{\circ} \mathrm{C}$, 

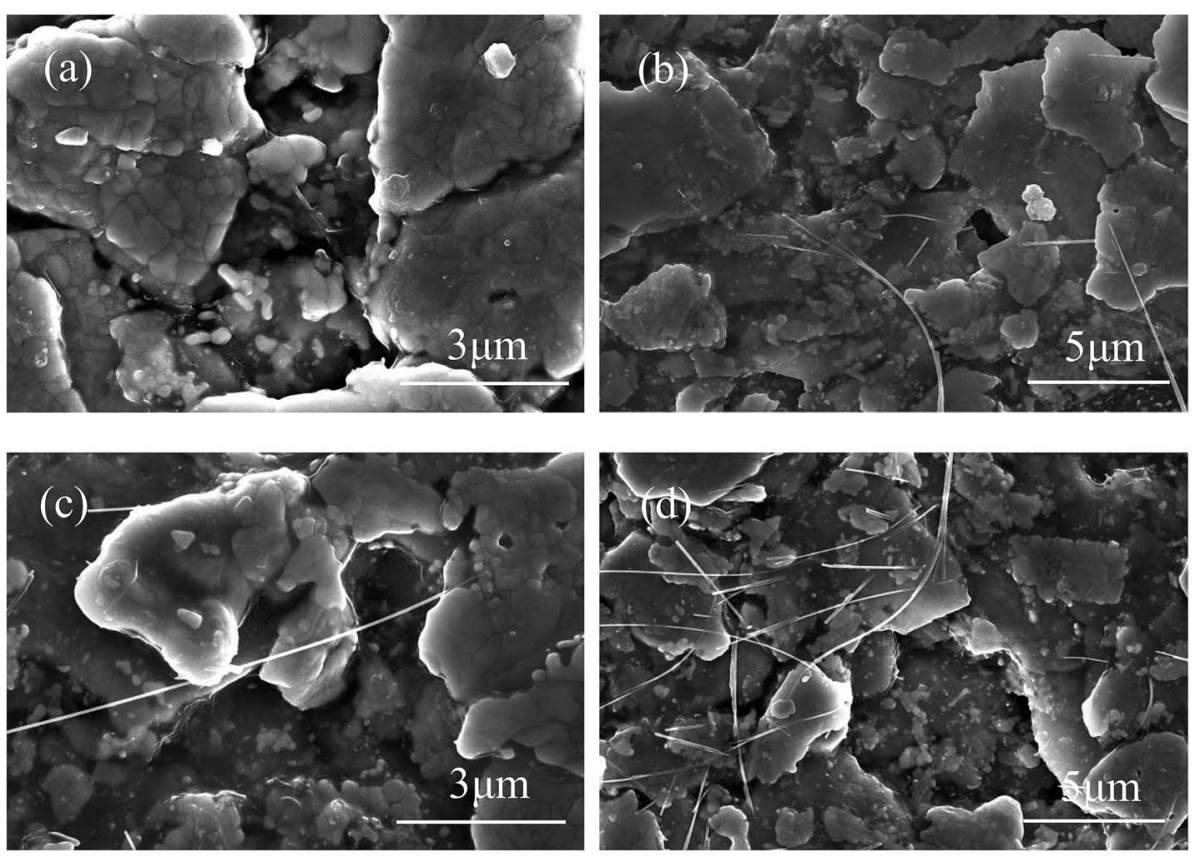

Fig. 6 FESEM images of silver-epoxy paste prepared by addition of ( $a$ and b) pristine MWCNTs and (c and d) Ag-NPs/MWCNT composites.

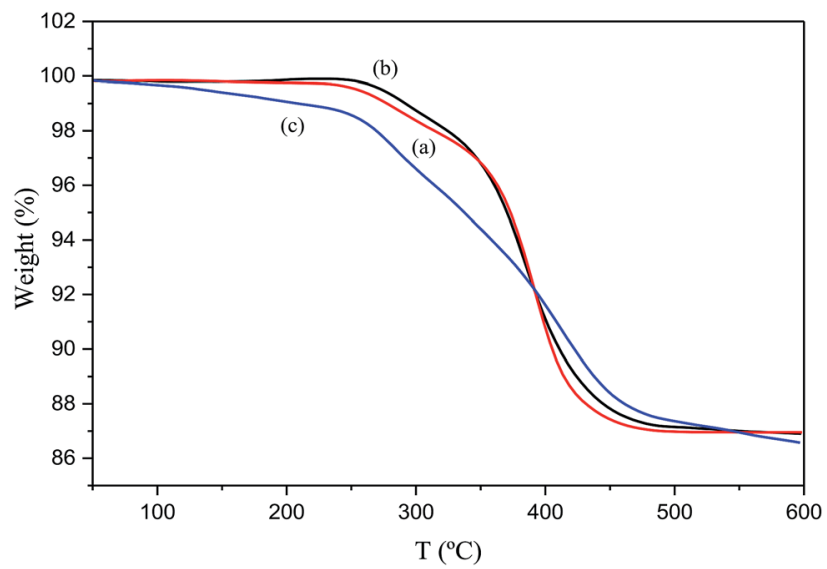

Fig. 7 TG curves of (a) silver-epoxy paste with added MWCNTs, (b) pristine silver-epoxy paste, and (c) silver-epoxy paste with added AgNPs/MWCNT composites.

the residual mass was estimated to be $86.901 \%$, and the mass change was $11.423 \%$. Fig. 7 (c) displays a significant weight loss from $313.0{ }^{\circ} \mathrm{C}$ with a residual mass of $94.656 \%$. At $599.5{ }^{\circ} \mathrm{C}$, the residual mass was estimated to be $82.933 \%$, and the mass change was $11.723 \%$. Compared to pristine silver-epoxy paste and silver-epoxy paste with added MWCNTs, the silver-epoxy paste with added Ag-NPs/ MWCNT composites revealed more weight loss. This might be assigned to the dispersion of MWCNTs modified by AgNPs in the paste matrix, which sintered Ag-NPs with Ag flakes together to form large numbers of contact points. These, in turn, were oxidized to silver oxide during heating. Silver oxide degraded at higher temperatures to yield pure silver, and MWCNTs also decomposed at high temperatures.

\subsection{Thermal conductivity}

3.3.1 Mechanism of thermal conduction. Incorporating a highly thermally conductive filler into a silver-epoxy paste is one of the most promising methods for improving its thermal conductivity, and the thermal conductivity is dominated by the filler. ${ }^{15}$ As shown in Fig. 8, phonon scattering can occur at the filler/matrix interface; the Ag-NPs/MWCNT composites and Ag flakes form a three-dimensional heat-conducting structure by

\section{Phonon transport}

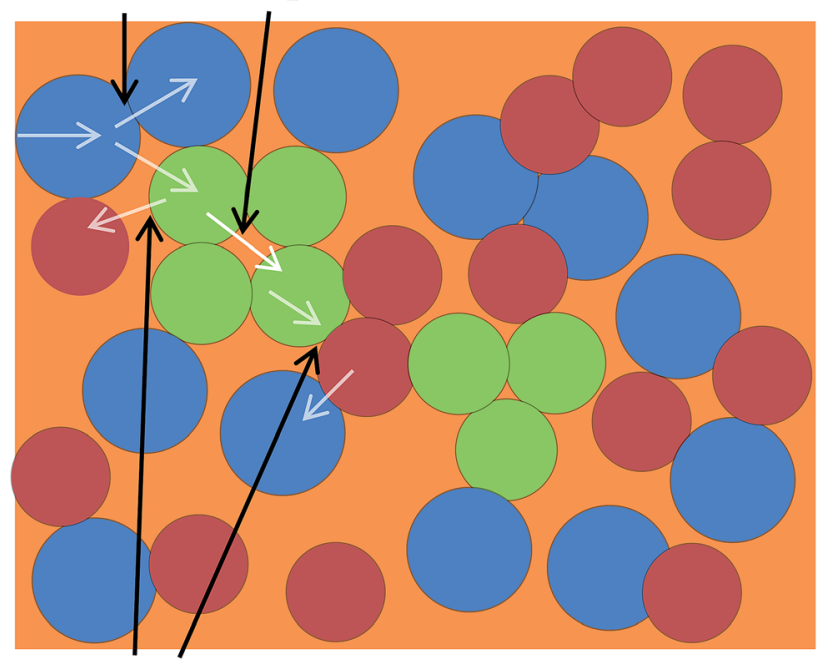

\section{Phonon scattering}

Fig. 8 Thermal conduction mechanism in silver-epoxy paste with added filler. The green balls represent Ag/MWCNT composites, the blue balls represent $\mathrm{Ag}$ flakes, and the red balls represent polymer atoms. 


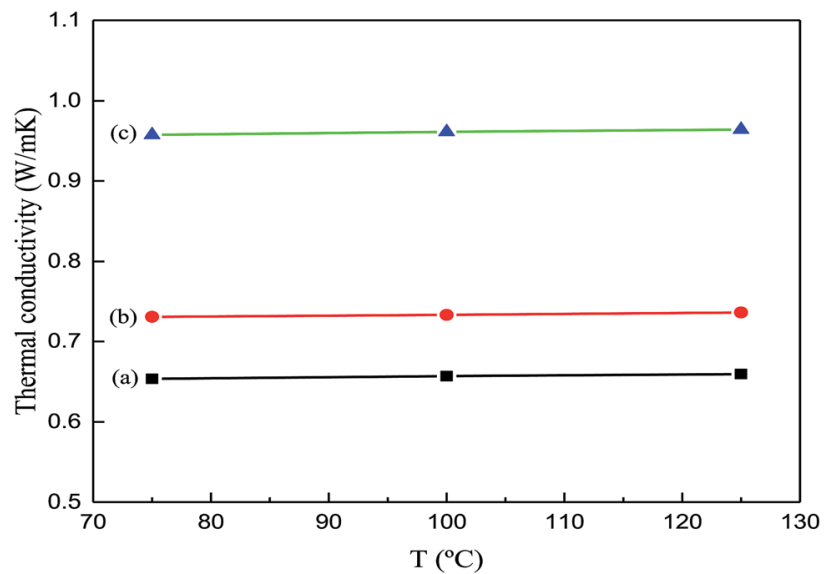

Fig. 9 Thermal conductivities of (a) silver-epoxy paste with added MWCNTs, (b) pristine silver-epoxy paste, and (c) silver-epoxy paste with added Ag-NPs/MWCNT composites.

low-temperature sintering bonding, forming a solid phonon transport junction and effective phonon transport pathway.

3.3.2 Thermal conductivity analysis. The thermal conductivities of the silver-epoxy pastes were investigated from 75 to $125^{\circ} \mathrm{C}$, and the thermal conductivity of the unfilled cured epoxy paste was about $0.25 \mathrm{~W} \mathrm{~m}^{-1} \mathrm{~K}^{-1}$. Fig. 9(a-c) illustrate the change in the thermal conductivities of the silver-epoxy paste with added MWCNTs, pristine silver-epoxy paste, and silverepoxy paste with added Ag-NPs/MWCNT composites. The thermal conductivity of the pristine paste was estimated to be $0.73 \mathrm{~W} \mathrm{~m}^{-1} \mathrm{~K}^{-1}$. Compared to the thermal conductivity of the pristine paste, the thermal conductivity of the paste with added MWCNTs was reduced to about $0.65 \mathrm{~W} \mathrm{~m}^{-1} \mathrm{~K}^{-1}$ due to the presence of MWCNTs in the paste with elevated inter-tube resistance. The latter would form a higher transmission barrier with the Ag flakes, increasing the interface thermal resistance and decreasing the thermal conductivity. ${ }^{32,33}$ The thermal conductivity of the paste with the added Ag-NPs/ MWCNT composites significantly improved when compared to that of the pristine paste $\left(0.96 \mathrm{~W} \mathrm{~m}^{-1} \mathrm{~K}^{-1}\right)$. This was attributed to the attachment of Ag-NPs on the MWCNT surfaces, which facilitated bonding at the interface with the $\mathrm{Ag}$ flakes during the curing process, forming a solid phonon transport junction and effective phonon transport pathway. The carrier transport barrier between the fillers was reduced, and the carrier interface transmission efficiency improved. Meanwhile, the Ag-NPs/MWCNT composites acted as a bridge between the $\mathrm{Ag}$ flakes to form numerous heat conduction paths. After absorption of heat by Ag-NPs on the MWCNT surfaces, it was transferred to MWCNTs. Since MWCNTs are thermal conductors with extremely high thermal conductivities, the heat was quickly absorbed.

\section{Conclusions}

Ag-NPs/MWCNT composites were prepared by functionalized ball milling of MWCNTs with SDBS as a dispersant and $\mathrm{C}_{6} \mathrm{H}_{8} \mathrm{O}_{6}$ as a reducing agent. Surface characterization revealed different
Ag-NPs uniformly attached to the MWCNT surfaces with high distribution densities; the average size of Ag-NPs was estimated to be $8.29 \mathrm{~nm}$. The as-obtained Ag-NPs/MWCNT composites were added to a silver-epoxy paste to form matrices with good wettability and dispersity. Compared to the pristine silver-epoxy paste, the matrices containing Ag-NPs/MWCNTs showed more weight loss with a mass change of $11.723 \%$ and increased thermal conductivity from 0.73 to $0.96 \mathrm{~W} \mathrm{~m}^{-1} \mathrm{~K}^{-1}$. This was attributed to the sintering bonding of Ag-NPs on the MWCNT surfaces at the interface during the curing process, which led to an effective phonon transport pathway. Ag-NPs/MWCNTs acted as a bridge between the $\mathrm{Ag}$ flakes, forming a thermal path. Overall, the Ag-NPs/MWCNT composites are promising for improving the properties of silver-epoxy pastes.

\section{Conflicts of interest}

There are no conflicts to declare.

\section{Acknowledgements}

This work was financially supported by the National Science Foundation of China (Grant No. 5177011044) and the Major Science and Technology Projects in Yunnan Province.

\section{References}

1 W. U. Haoyi, S. Chiang, W. Han, Y. Tang, F. Kang and C. Yang, Compos. Sci. Technol., 2014, 99, 109-116.

2 L. Rivière, A. Lonjon, E. Dantras, C. Lacabanne, P. Olivier and N. R. Gleizes, Eur. Polym. J., 2016, 85, 115-125.

3 D. Suh, C. M. Moon, D. Kim and S. Baik, Adv. Mater., 2016, 28, 7220-7227.

4 K. Kim, B. Park, H. Kim, H. Lee and S. Jung, Curr. Appl. Phys., 2015, 15, S36-S41.

5 J. R. Choi, K. Y. Rhee and S. J. Park, Composites, Part B, 2015, 80, 379-384.

6 G. Yuan, A. M. Marconnet, X. Rong, S. Maruyama and K. E. Goodson, IEEE Trans. Compon., Packag., Manuf. Technol., 2013, 3, 1524-1532.

7 Y. Zhou, Z. Luo, Z. Xiao and L. Feng, Mater. Lett., 2016, 182, 323-327.

8 M. Seo, J. S. Kim, J. G. Lee, S. B. Kim and M. K. Sang, Thin Solid Films, 2016, 616, 366-374.

9 Y. Zhao, P. Mumby-Croft, S. Jones, A. Dai, Z. Dou, Y. Wang and Q. Feng, Applied Power Electronics Conference \& Exposition, IEEE, 2017, pp. 3091-3094.

10 I. Cesarino, V. Cesarino, F. C. Moraes, T. C. R. Ferreira, M. R. V. Lanza, L. H. Mascaro and S. A. S. Machado, Mater. Chem. Phys., 2013, 141, 304-309.

11 Y. Zhang, J. R. Choi and S. J. Park, Polymer, 2018, 143, 1-9. 12 L. Yi, K. A. Watson, M. J. Fallbach, G. Sayata, J. G. Smith, D. M. Delozier, C. Wei, R. E. Crooks and J. W. Connell, ACS Nano, 2009, 3, 871-884.

13 M. Li, Y. Xiao, Z. Zhang and J. Yu, ACS Appl. Mater. Interfaces, 2015, 7, 9157-9168. 
14 C. H. Kuo and H. M. Huang, J. Therm. Anal. Calorim., 2011, 103, 533-542.

15 Y. Zhang, Y. Heo, Y. Son, I. In, K. An, B. Kim and S. Park, Carbon, 2019, 142, 445-460.

16 Y. Zhang, J. R. Choi and S. J. Park, Composites, Part A, 2018, 143, 1-9.

17 Q. Zhao, M. Xie, Y. Liu and J. Yi, Appl. Surf. Sci., 2017, 409, 164-168.

18 H. Pal and V. Sharma, Trans. Nonferrous Met. Soc. China, 2015, 25, 154-161.

19 W. M. Daoush and S. H. Hong, J. Exp. Nanosci., 2013, 8, 742751.

20 S. Chen, Y. Wei, L. Zou, H. Lu, Y. Xu, J. Hua, H. Sun, X. Peng and B. Liu, Appl. Organomet. Chem., 2016, 31, 1-7.

21 Y. Yusof, M. I. Zaidi and M. R. Johan, J. Nanomater., 2016, 2016, 6141496.

22 A. K. Singh, B. P. Panda, S. Mohanty, S. K. Nayak and M. K. Gupta, J. Mater. Sci.: Mater. Electron., 2017, 28, 89088920 .

23 K. Kim, K. S. Kim, G. Kim and S. Baeck, Curr. Appl. Phys., 2012, 12, 36-39.

24 L. Chen, H. Xie and W. Yu, J. Mater. Sci., 2012, 47, 5590-5595.
25 K. Wusiman, H. Jeong, K. Tulugan, H. Afrianto and H. Chung, Int. Commun. Heat Mass Transfer, 2013, 41, 28-33.

26 V. H. Nguyen and J. J. Shim, Synth. Met., 2011, 161, 20782082.

27 R. Sadri, G. Ahmadi, H. Togun, M. Dahari, S. N. Kazi, E. Sadeghinezhad and N. Zubir, Nanoscale Res. Lett., 2014, 9, 151.

28 T. J. Choi, S. P. Jang and M. A. Kedzierski, Int. J. Heat Mass Transfer, 2018, 122, 483-490.

29 X. Ling, Y. Wei, L. Zou and X. Su, Colloids Surf., A, 2014, 443, 19-26.

30 V. Á. Jarmila, M. K. Robert, S. Petr, I. Í. Markéta, K. Natalia, H. I. Martina, M. U. Í. Matej and O. Mária, Molecules, 2012, 17, 13157-13174.

31 W. Zhao, H. Wang, X. Qin, X. Wang, Z. Zhao, Z. Miao, L. Chen, M. Shan, Y. Fang and Q. Chen, Talanta, 2010, 80, 1029-1033.

32 A. M. Marconnet, Y. Namiko, M. A. Panzer, B. L. Wardle and K. E. Goodson, ACS Nano, 2011, 5, 4818-4825.

33 Z. Talaei, A. R. Mahjoub, A. M. Rashidi, A. Amrollahi and M. E. Meibodi, Int. Commun. Heat Mass Transfer, 2011, 38, 513-517. 